\title{
Uniaxially Tensile Strained Accumulation-Mode Gate-All-Around Si Nanowire nMOSFETs
}

\author{
Mohammad Najmzadeh, Didier Bouvet, Wladek Grabinski, Adrian M. Ionescu \\ Nanoelectronic devices lab., Swiss Federal Institute of Technology (EPFL), CH-1015 Lausanne, Switzerland \\ phone: +41 21693 5633, email: mohammad.najmzadeh@epfl.ch
}

In this work we report an experimental study on accumulation-mode (AM) gate-all-around (GAA) nMOSFETs based on silicon nanowires with uniaxial tensile strain. Their electrical characteristics are studied from room temperature up to $\sim 400 \mathrm{~K}$ and carrier mobility, flat-band and threshold voltages are extracted and investigated.

Recently, highly single-type doped devices such as accumulation-mode (AM) and junctionless (JL) FETs have been proposed as straightforward switch architectures able to eliminate some of the limitations of nano-scale FETs such as the ultra-abrupt junctions and related processing challenges, allowing to fabricate even shorter channel devices [1-3]. Achieving a high $\mathrm{I}_{\mathrm{on}}$ in a heavily doped channel is an engineering challenge since the carrier mobility in heavily doped devices is limited by ionized impurity scattering. In this paper we evaluate the impact of a local uniaxial tensile strain engineering method by local bending of nanowires to improve the mobility in highly doped AMOSFETs, without affecting their $\mathrm{I}_{\mathrm{on}} / \mathrm{I}_{\text {off }}$.

Figure 1 represents the process flow to make dense array $(\sim 8 \mathrm{NW} / \mu \mathrm{m})$ of deeply scaled sub-50 nm wide/thick $\mathrm{Si}$ nanowires on a Silicon-On-Insulator substrate. A sacrificial oxidation was formed in the presence of silicon nitride hard mask to shrink further the width of the Si nanowires, round the possible sharp corners as well as accumulate uniaxial tensile stress in the channel after stripping the hard mask and suspending the channel [4]. The gate stack includes $5 \mathrm{~nm} \mathrm{HfO} \mathrm{HLD}_{2}$ ALposition, RTA and afterward, $20 \mathrm{~nm}$ TiN deposition by sputtering to accumulate further uniaxial tensile stress in the suspended nanowires [5], causes local buckling of the suspended nanowires and induces clearly $>1.5 \mathrm{GPa}$ uniaxial tensile stress in the channel, measured from the arc length of the buckled NWs.

The electrical characterization was carried out at different temperatures using a Cascade prober and a HP 4155B Semiconductor Parameter Analyzer. Figure 2 depicts the $I_{D}-V_{G}$ and $I_{D}-V_{D}$ characteristics of a $2 \mu \mathrm{m}$ long channel AMOSFET, including an array of 10 parallel Si nanowires, at $298 \mathrm{~K}$. According to [6] and as described in Figure 3, the flat-band condition in an AMOSFET is pretty close to the threshold voltage and the accumulation layer will be created after reaching the flat-band condition while a heavily doped JL MOSFET, due to having the main current at the middle of the channel above threshold voltage, is mainly operating below the flat-band voltage and the flat-band condition will be reached while reaching the density of carriers on the surface to the doping concentration. Note that in the subthreshold region $\left(\mathrm{V}_{\mathrm{G}}<\mathrm{V}_{\mathrm{TH}}\right)$ the conduction in both AMOSFET and JLFET is in the bulk of the NW.

The threshold voltage, $\mathrm{V}_{\mathrm{TH}}$, of the AMOSFET was extracted using the transconductance change (TC) method [7] which is quasi-independent of device series resistance and does not require the use of any accurate analytical model. Interestingly, as shown in Figure 4, the TC peak is appearing almost at the expected (theoretical) threshold voltage from the linear region of the $\mathrm{I}_{\mathrm{D}}-\mathrm{V}_{\mathrm{G}}$ curve. By considering the current in the accumulation channel as the main dominant current in the GAA nanowire and using an earlier developed analytical model in [8], the flat-band voltage was extracted using the $\mathrm{I}_{\mathrm{D}} / \mathrm{gm}^{0.5}$ method [9] independent of series resistances and mobility attenuation factor and the results were plotted in Figure 4, providing systematically higher value than $\mathrm{V}_{\mathrm{TH}}$ [6].

The transfer characteristics of the AMOSFET at 298-398 K are reported in Figure 5. The low field electron mobility at different temperatures was extracted using the slope of the $\mathrm{I}_{\mathrm{D}} / \mathrm{gm}^{0.5} \mathrm{vs}$. $\mathrm{V}_{\mathrm{GS}}$ in the linear region $\left(\mathrm{V}_{\mathrm{DS}}=100 \mathrm{mV}\right)$, minimizing the influence of series resistances and mobility attenuation factor and plotted in Figure 6. As Figure 6 shows, the electron mobility in highly doped AMOSFET is reducing as $\mathrm{T}^{-0.97}$ which is lower than for intrinsic or low $\mathrm{n}$-doped $\mathrm{Si}$ and in agreement with prior reports [10], being explained by the dominant role of ionized impurity scattering in highly doped Si MOSFETs. Similar lower dependence on temperature is expected in JL FETs.

We report a threshold voltage reduction coefficient with temperature of $-0.92 \mathrm{mV} / \mathrm{K}$, a flat-band voltage coefficient with temperature of $-1.73 \mathrm{mV} / \mathrm{K}$ and, finally, a subthreshold slope degradation of $-0.43 \% / \mathrm{K}$ or $-0.456 \mathrm{mV} / \mathrm{dec} . \mathrm{K}$ (with a $106 \mathrm{mV} / \mathrm{dec}$. subthreshold slope at $298 \mathrm{~K}$ ), as reported in Figure 7.

As a conclusion, we have demonstrated the first AM gate-all-around nMOSFETs on SOI substrates with electron mobility enhanced by process-induced uniaxial tensile strain and reported their performance from $298 \mathrm{~K}$ to $398 \mathrm{~K}$.

This work was supported by Swiss National Foundation (SNF). Thanks to CIME-EPFL for SEM observation.

[1] J.-P. Colinge et al., Nature Nano. (2010). [2] J.-P. Colinge et al., Jpn. J. Appl. Phys., vol. 48, (2009). [3] J. Wu et al., ECS Transactions, vol. 33 (2010). [4] M. Najmzadeh et al., Microelec. Eng. (2009). [5] N. Singh et al., IEEE EDL (2007). [6] A. Nazarov et al., Springer, $1^{\text {st }}$ ed. (2011). [7] H.-S. Wong et al., SSE (1987). [8] J.-P. Colinge et al., IEEE TED, vol. 37, (1990). [9] G. Ghibaudo, Electronics Letters (1988). [10] S. Reggiani et al, IEEE TED, Vol. 49, 2002. 
(100) Unibond 4" SOI, intrinsic p-type Channel ion imp/ann. (Phosph., $1 \mathrm{e} 18 \mathrm{~cm}^{-3}$ ) LPCVD silicon nitride hard mask dep.

Active layer pattern by e-beam lithography Sacrificial dry oxidation + strip hard mask BOX etching to suspend the Si nanowires

High-k/metal gate stack deposition

Gate pattern - optical lithography and etch

S/D imp./anneal (Phosph., $22 \mathrm{e}^{2} \mathrm{~cm}^{-3}$ )

Metallization (AlSi-1\%)

(a)

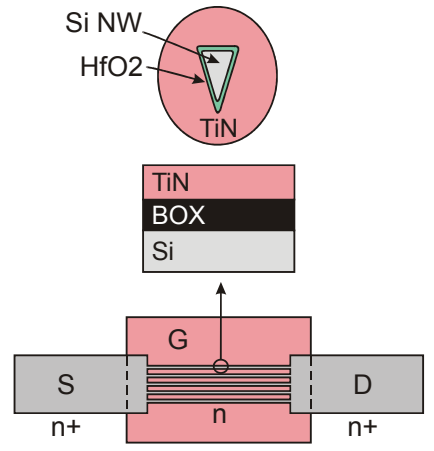

(b)

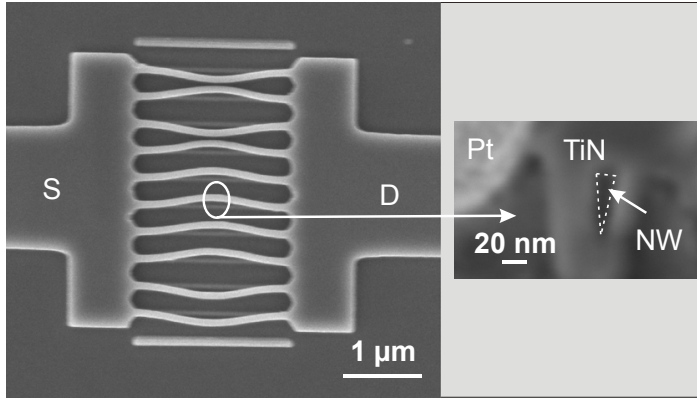

(c)

Fig. 1. Process flow to make GAA Si nanowire AMOSFET using a top-down Si NW platform (a), the schematic of the GAA Si NW AMOSFET (b), the SEM picture of an array of strained Si NWs after the gate stack step and the cross-section of a suspended GAA NW AMOSFET (right).

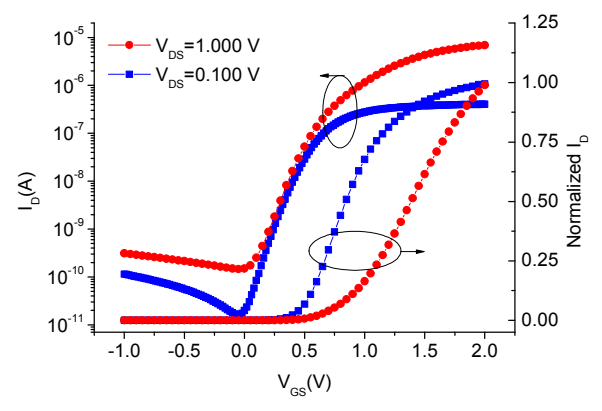

(a)

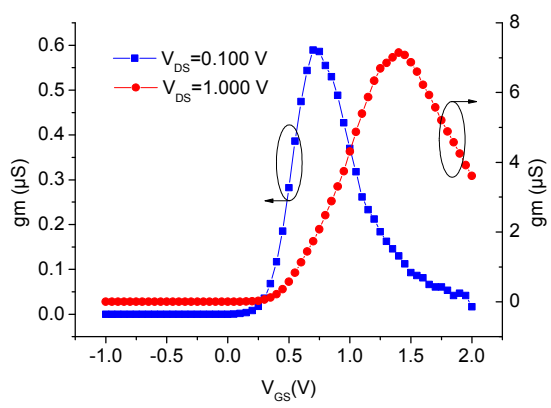

(b)

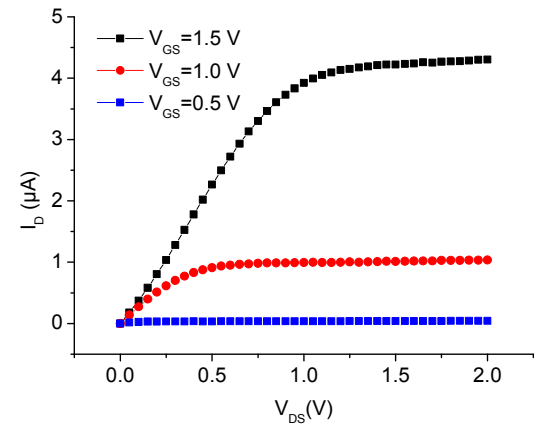

(c)

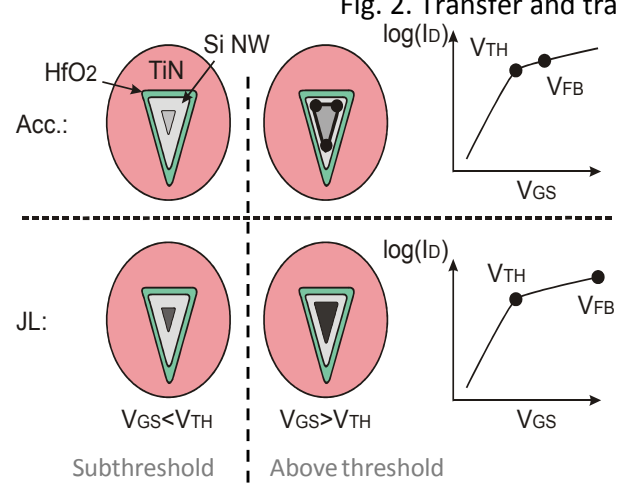

Fig. 3. Depiction of conduction path in GAA accumulation and junctionless MOSFETs vs. $V_{G S}$.

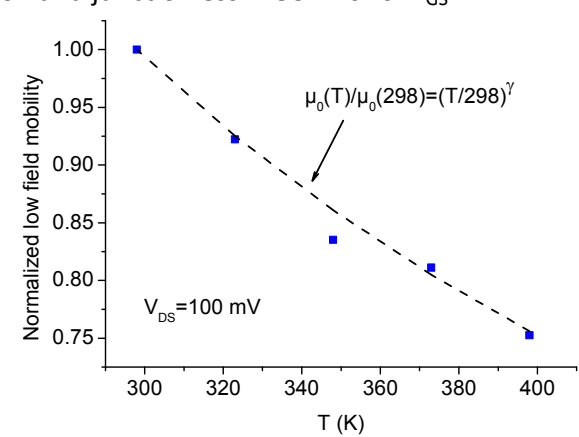

Fig. 6. Normalized low field electron mobility dependence on temperature for a GAA strained AMOSFET. The extracted $\gamma$ is -0.966 .

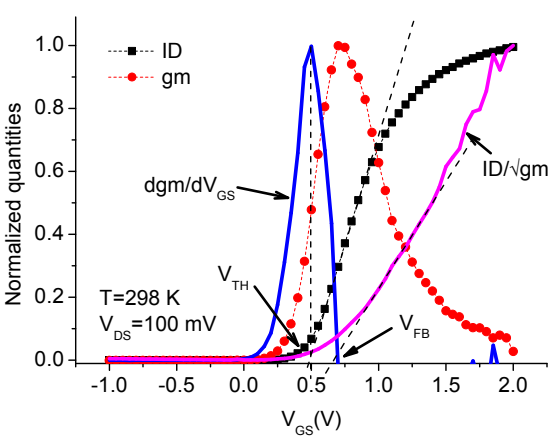

Fig. 4. Extraction of $V_{T H}$ and $V_{F B}$ of a $G A A$ AMOSFET using TC and ID/gm ${ }^{0.5}$ methods.

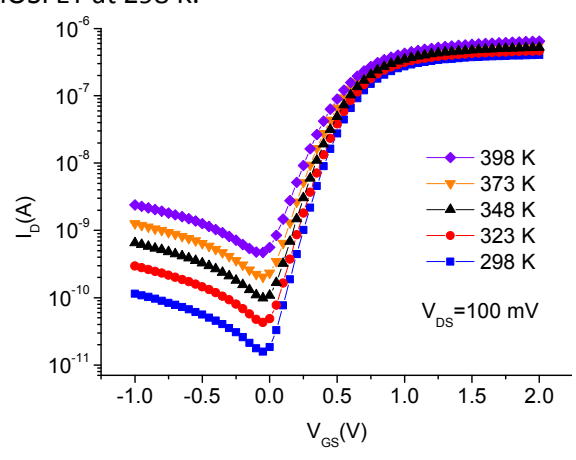

Fig. 5. Transfer charac. of a GAA strained AMOSFET at different temperatures.

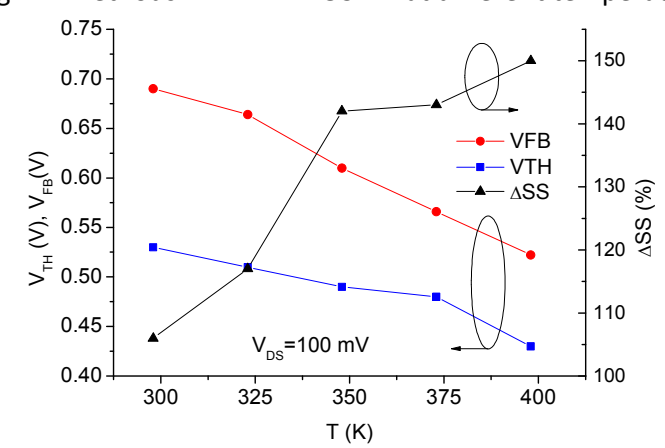

Fig. 7. Variation of extracted threshold voltage, flat-band voltage and subthreshold slope of a GAA strained AMOSFET with temperature. 